\title{
Signal processing on waveform data from the Eyesafe Ladar Test-bed (ELT)
}

\author{
Kevin D. Neilsen, Scott E. Budge, and Robert T. Pack \\ Center for Advanced Imaging Ladar, Utah State University, Logan, UT 84322-4170
}

\begin{abstract}
The Eyesafe Ladar Test-bed (ELT) is a raster scanning, single-beam, energy-detection ladar with the capability of digitizing and recording the return pulse waveform at $2 \mathrm{GHz}$ in the field for off-line 3D point cloud formation research in the laboratory. The ELT serves as a prime tool in understanding the behavior of ladar waveforms. Signal processing techniques have been applied to the ELT waveform in an effort to exploit the signal with respect to noise reduction, range resolution improvement, and ability to discriminate between two surfaces of similar range.

This paper presents a signal processing method used on the ELT waveform. In the processing, three deconvolution techniques were investigated-the Wiener filter, Richardson-Lucy deconvolution, and a new method that synthesizes the surface response using least squares minimization. Range error and range resolution are reported for these methods.
\end{abstract}

Keywords: VISSTA ELT, ladar, waveform, deconvolution, surface response

\section{INTRODUCTION}

Digitized waveforms have led to the capability to explore signal processing techniques that exploit the information in the ladar pulse. Given measured parameters for signal and noise, optimal filtering can be used for noise reduction. This provides the ability to improve range error, which is defined as the standard deviation of the measured range from the true range. ${ }^{1}$ False alarms and dropouts are also minimized because the user can view the waveform and set threshold levels according to the noise and signal to maximize performance.

Not only do these systems improve traditional performance metrics, but also add new capabilities that present information beyond only a simple range measurement. One of the most common discrimination methods for digitized waveforms is Gaussian decomposition. ${ }^{2-6}$ It provides the user with an accurate measurement of range and amplitude as well as a measurement of changes in the pulse width.

Gaussian decomposition works by assuming that a signal is made up of a sum of individual returns modelled by Gaussian pulses. If the number of returns that make up the signal is known, the signal can be readily decomposed; however, if the number of returns is unknown, the algorithm can be run iteratively to find the number of returns that present the lowest error in the modelled signal. ${ }^{7}$ Gaussian decomposition has been performed using the Levenberg-Marquardt algorithm as well as the expectation maximization (EM) algorithm. ${ }^{2}$ The Levenberg-Marquardt algorithm finds the least squares fit to the pulses, and the solution using the EM algorithm converges to the maximum likelihood solution. ${ }^{8}$

A more recently developed method treats the received waveform as a blurred version of the desired response. ${ }^{5,9,10}$ The blur, caused by the temporal waveform of the transmitted pulse, is convolved with the response due to the surface to obtain the measured waveform. The goal is to deconvolve the transmitted pulse out of the received waveform in order to obtain a clear surface response of the target.

It has been shown by Jutzi and Stilla that obtaining the surface response has made it possible to detect two surfaces offset by $15 \mathrm{~cm}$ with a single ladar shot using a $5 \mathrm{~ns}$ full width half max (FWHM) transmitted pulse. ${ }^{5}$ Data in these experiments was collected at a sampling rate of $20 \mathrm{GHz}$ (over 100 times Nyquist) for specular surfaces at a range of $100 \mathrm{~m}$. Because of the high sample rate and conditions used to maximize the SNR, the authors concluded that under such conditions, digitized waveforms greatly improve the ability to resolve two surfaces. However, they did not experiment on data collected with a lower sample rate that could be sustained to collect high density point clouds using a large range gate. 
The purpose of this paper is to present signal processing techniques that can be used on a waveform digitizing ladar with a practical sample rate for forming 3D point clouds. Section 2 provides an introduction to the system used to collect data. Section 3 outlines the techniques used for data processing. Section 4 contains the experiments used to compare methods, and conclusions are given in Section 5.

\section{THE ELT}

Shown in Fig. 1, the Vehicle Integrated Sensor Suite for Targeting Applications (VISSTA) van was developed to provide a mobile sensor platform for multiple modes of data collection. ${ }^{11}$ The ELT, mounted in the VISSTA van, has a single sensor flying spot scanner capable of custom scanning patterns. The ELT and an IR camera sit inside a movable turret on top of the van. To allow the operator to observe the field of view of the ELT scanner, the ELT transmitter contains an eyesafe laser that is mounted co-axially with a color digital camera. This also enables $3 \mathrm{D}$ texel images to be formed by fusing the camera image with the $3 \mathrm{D}$ point cloud. ${ }^{12}$

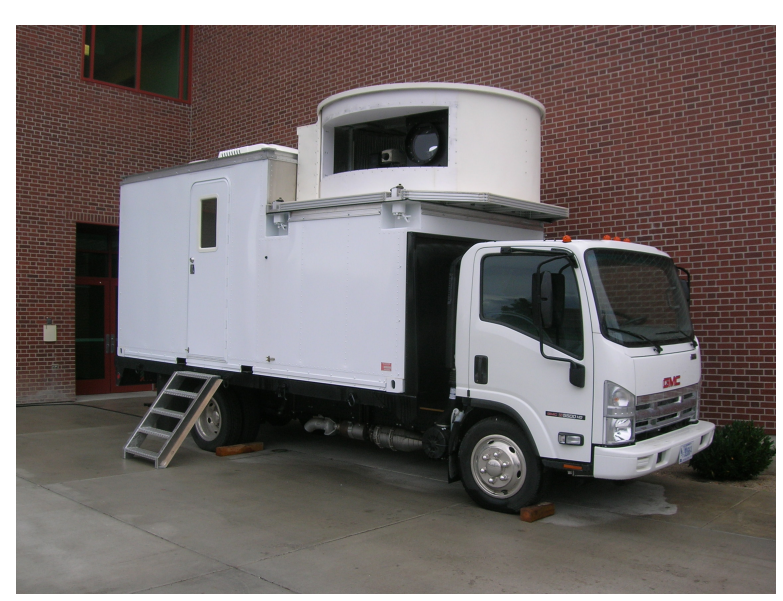

(a)

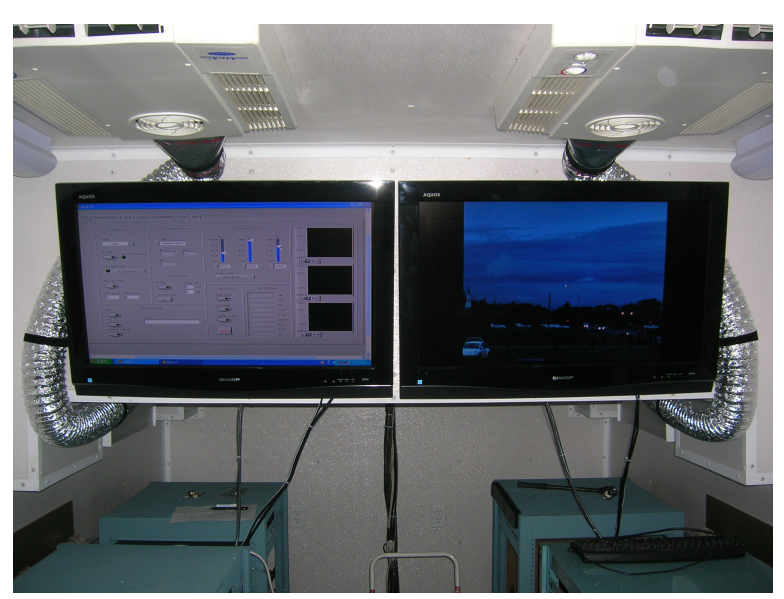

(b)

Figure 1: The VISSTA van. (a) The ELT aperture can be seen at the right side in the turret behind the cab. (b) Displays used for system control and display of sensor data.

The ELT digitizes the return pulse waveform at $2 \mathrm{GHz}$ for offline data analysis. The laser pulse width is $1.5 \mathrm{~ns}$ at FWHM, resulting in waveforms sampled at two times the Nyquist frequency. The ELT is capable of scanning a $4^{\circ} \times 4^{\circ}$ field of view at up to a $100 \mathrm{kHz}$ pulse rate. To provide flexibility in scene acquisition, the range gate of the digitizer can be set up to $500 \mathrm{~m}$ for a $20 \mathrm{kHz}$ pulse rate.

\section{DATA PROCESSING}

The data acquisition model for the ladar receiver is given in Fig. 2. Separate electronics are used to detect the transmitted pulse as it exits the sensor and the return pulse from the target, and are of the same design. Different electronics transfer functions $(H(s))$ are used in modelling the digitized exiting pulse and the return pulse due to slight mismatches between the receiver and transmitter electronics.

The input to the system is the Dirac delta function $\delta(t)$, representing the ideal transmitted pulse shape. First, the input is convolved with the laser pulse transfer function $P(s)$ to create the actual transmitted laser pulse waveform. The pulse then travels into the environment where it interacts with the target. This is viewed as the convolution of the pulse with the target surface response $S(s)$. Backscatter, solar background noise, dark current noise, and noise in the receiver electronics are lumped together as additive white noise $n_{t}(t)$. Amplitude dependent (multiplicative) shot noise from the avalanche photodiode (APD) is added to the input to the electronics. The signal then proceeds through the receiver electronics $H(s)$. Finally, quantization and measurement noise $n_{q}(n)$ in the A/D converter is added to obtain the stored waveform $r(n)$. A more complete model of the receiver electronics is given in. ${ }^{13}$ 


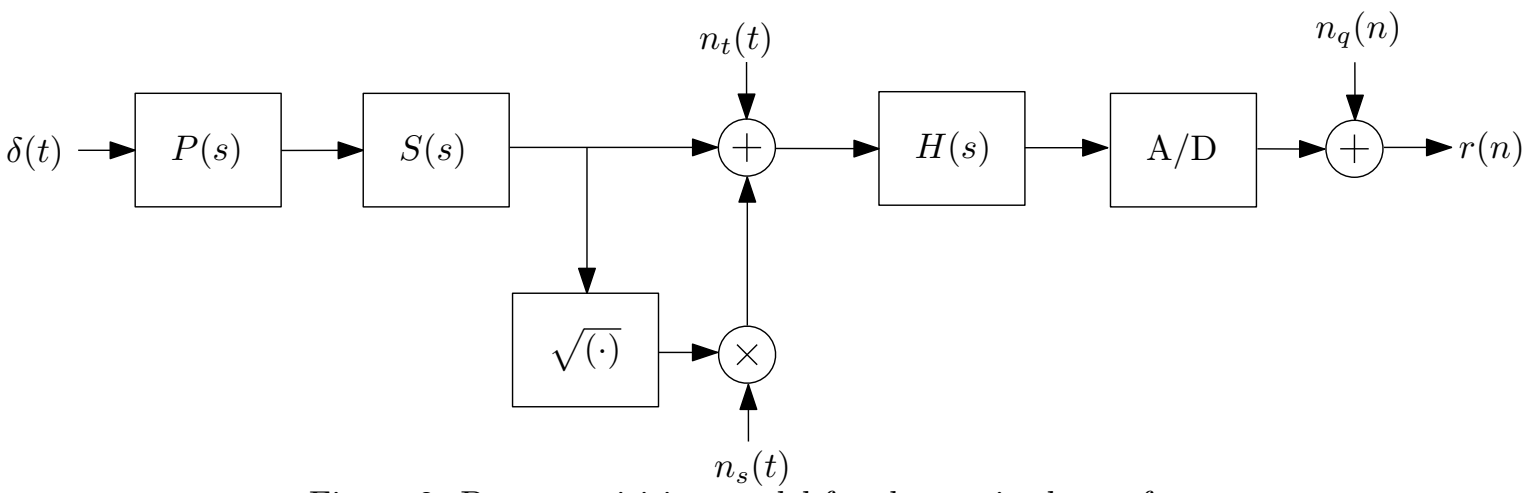

Figure 2: Data acquisition model for the received waveform.

Due to the difficulty in obtaining an estimate of the signal dependent shot noise, the model was simplified by assuming it can be modelled as additive white noise with variance proportional to the average value of the pulse waveform. This leads to the frequency-domain sampled signal

$$
R(f)=P(f) S(f) H(f)+N(f) H(f)+N_{q}(f),
$$

where

$$
\begin{gathered}
N(f)=N_{t}(f)+\sqrt{\overline{p_{s}(t)}} N_{s}(f), \\
p_{s}(t)=p(t) * s(t),
\end{gathered}
$$

$\overline{p_{s}(t)}$ is the time average of $p_{s}(t)$ during the duration of the pulse,

$f$ is the normalized discrete frequency,

$N_{t}(f), N_{s}(f)$ are white noise, and

$p(t)$ and $s(t)$ are the inverse Laplace transforms of $P(s)$ and $S(s)$ respectively.

The goal of processing the data is to recover the discrete-time surface response $S(z)$ from the received waveform $r(n)$. For the ELT, this is achieved by the process of filtering, interpolation, deconvolution, and range discrimination as shown in Fig. 3, where $z$ is used to denote a discrete-time transfer function. The remainder of this section describes each process used to obtain the surface response.

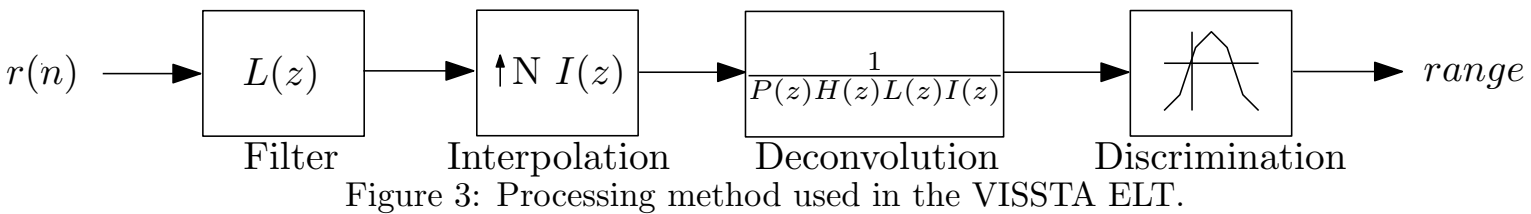

\subsection{Lowpass (matched) filtering}

The first step in processing the captured waveform $r(n)$ is to lowpass filter the signal. This is done to (1) approximate a matched filter, and (2) provide a regularization to the deconvolution process, which is known to be an ill-posed problem.

Before deconvolving the signal, it is desirable to be able to detect potential return pulses and process only those parts of the signal. The ability to detect pulses is improved if the signal is filtered by a matched filter $L(z)$ to reduce false alarms and improve the signal-to-noise ratio (SNR). This filter is approximated by a lowpass filter with a bandedge located at the highest frequency in the transmitted pulse spectrum. It is significantly easier to detect return pulses in the resulting signal. An example of the improvement is illustrated in Fig. 4. Also, since 


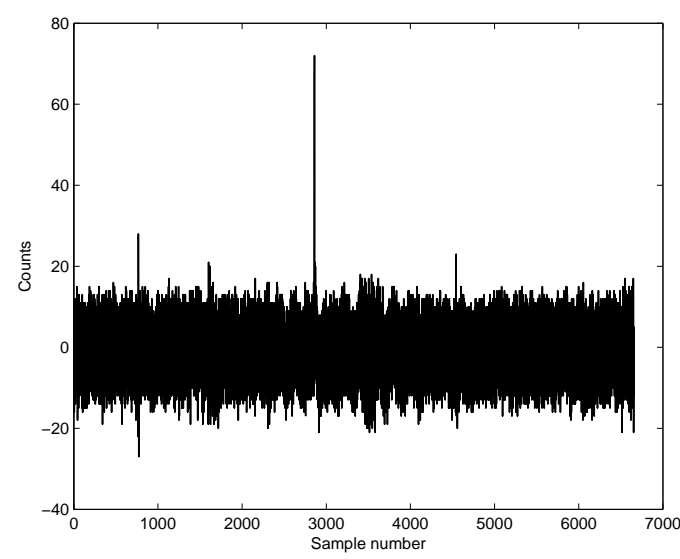

(a)

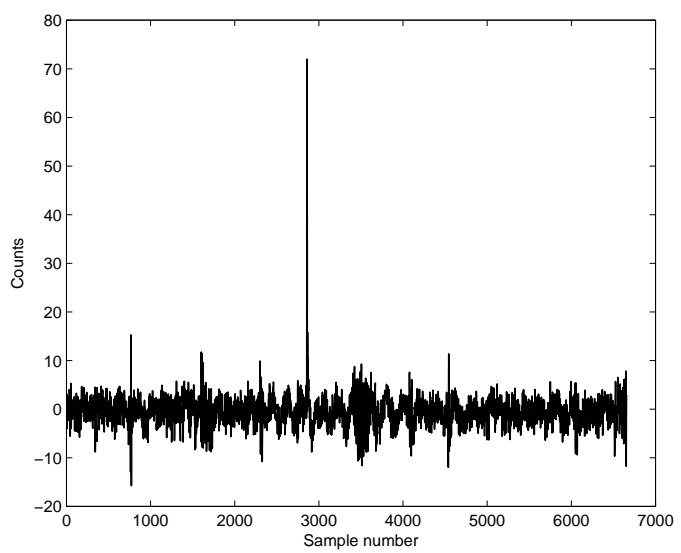

(b)

Figure 4: Waveform of a shot at a sufficient range to show high noise levels. (a) The waveform before the matched filter. (b) The waveform after the matched filter.

the pulse spectrum only extends to half of the foldover frequency, a significant amount of the white noise signal $N(f)$ is in the stopband of the matched filter and is therefore reduced by the filter.

The transfer functions $P(z)$ and $H(z)$ are lowpass in nature, and the inverse of these functions has a tendency to boost high-frequency noise. Deconvolution in the presence of noise can result in a poor estimate of the surface response $S(z)$. Therefore, reduction of high frequency noise by the matched filter also improves the behavior of the deconvolution estimate.

\subsection{Interpolation}

The ELT digitizes data at $2 \mathrm{GHz}$ which corresponds to $7.5 \mathrm{~cm} /$ sample in range. Relative to the desired measurement accuracy, $7.5 \mathrm{~cm} /$ sample is very coarse and requires interpolation to a sample rate that is N-times larger. The sampling factor $\mathrm{N}$ is typically set to 10 which decreases the sample time to $7.5 \mathrm{~mm} / \mathrm{sample}$. An example of a pulse interpolated by a factor of 10 is given in Fig. 5 .

Interpolation by a factor of $\mathrm{N}$ is performed by first upsampling the signal i.e. inserting $\mathrm{N}-1$ zeros between every sample in the signal. It can be shown that upsampling a signal causes the signal's spectrum to be compacted by a factor of $\mathrm{N}$ such that a frequency $f_{0}$ maps to $\frac{f_{0}}{N} \cdot{ }^{14}$ An artifact of the frequency compaction is that spectral copies of the original pulse are centered at $\mathrm{f}=\frac{1}{N}, \frac{2}{N}, \frac{3}{N} \ldots$ in normalized frequency. This is due to the periodic nature of sampled data. Next, the signal of interest is obtained by filtering the signal with a linear phase low pass filter, $I(z)$, that has a bandwidth of $\frac{1}{2 N}$ (normalized frequency). This eliminates the spectral copies and leaves only the interpolated signal.

If an interpolated version of $L(z)$ were used as the interpolation filter, it would not require the use of a filter before interpolation. However, applying $L(z)$ before interpolation obtains the same result but with a lower order filter. Due to the frequency compaction, an interpolation filter that produces results equivalent to those of $L(z)$ would require a transition band that is $\mathrm{N}$ times steeper than $L(z)$. For this reason, $L(z)$ is used before interpolation, permitting an interpolation filter with a more gradual transition band and hence a lower order filter.

\subsection{Deconvolution}

As the laser pulse travels to and from the scene, the return waveform is modified as it hits multiple surfaces at various angles within the beam footprint. ${ }^{5}$ Deconvolution allows us to determine the surface response of the target and use it to potentially obtain an estimate of the range to multiple surfaces within the beam footprint. Applying the lowpass filters $L(z)$ and $I(z)$ to $(1)$ leads to 


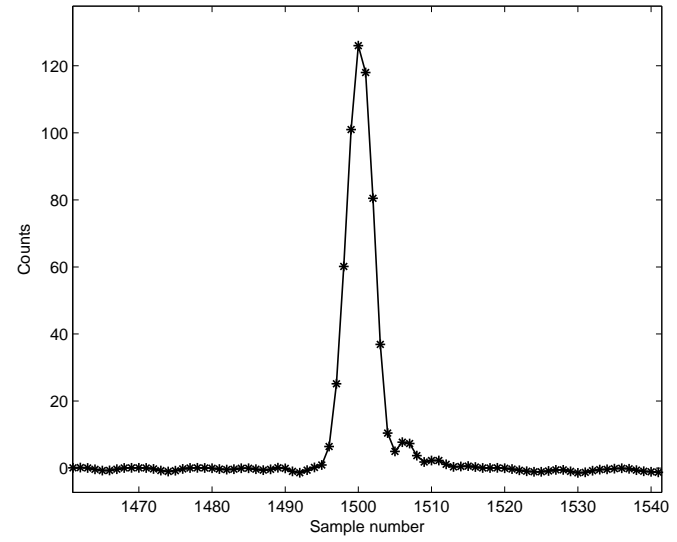

(a)

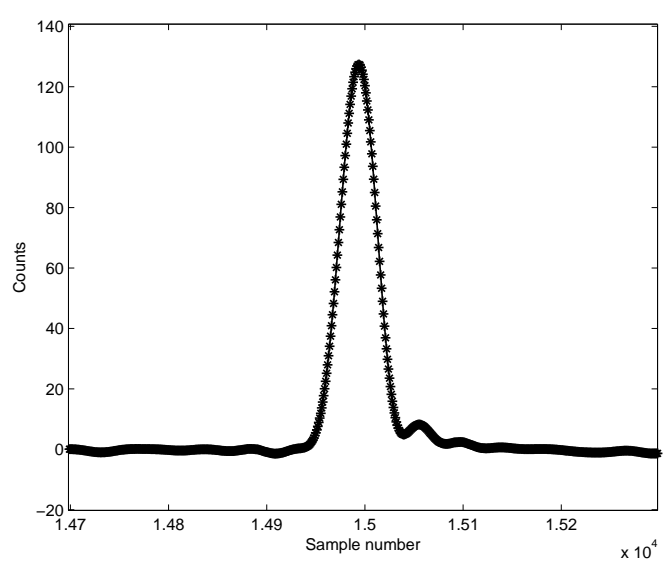

(b)

Figure 5: Waveform of interpolated pulse. (a) No interpolation. (b) Interpolation by a factor of 10.

$$
R(z) L(z) I(z)=\left(P(z) S(z) H(z)+N(z) H(z)+N_{q}(z)\right) L(z) I(z),
$$

which can be rearranged to obtain the target surface response

$$
S(z)=\frac{\tilde{R}(z)}{\tilde{H}(z)}+\tilde{N}(z)
$$

where

$$
\begin{gathered}
\tilde{R}(z)=R(z) L(z) I(z) \\
\tilde{H}(z)=P(z) H(z) L(z) I(z),
\end{gathered}
$$

and

$$
\tilde{N}(z)=\frac{N(z)}{P(z)}+\frac{N_{q}(z)}{P(z) H(z)} .
$$

Deconvolution is possible only if $\tilde{R}(z)$ and $\tilde{H}(z)$ are known and effects from $\tilde{N}(z)$ can be minimized. $\tilde{R}(z)$ is the measured waveform after filtering and interpolation. To obtain $\tilde{R}(z)$, the locations of the returns in the signal are found and $r(n)$ is segmented into noise and signal segments. Each signal segment can then be processed individually.

$\tilde{H}(z)$ (the blur function) is obtained by holding the scanner stationary while aimed at a flat surface oriented normal to the ELT, and averaging many returns together. Averaging nearly eliminates noise and provides an accurate estimate of the blur function. Once the blur function is obtained, it may be used for any experiment that shares the same receiver electronics characteristics. Alternatively, the transmitted pulse for each shot could be used to estimate the blur function, ${ }^{5}$ but noise on each transmitted pulse waveform, combined with differences in the electronics in the ELT used in the capture of the transmitted pulse, produce a poorer estimate of the blur in the received waveforms than the averaging method used.

Although many deconvolution techniques exist, three were tested with the VISSTA ELT. These are the Wiener filter, the Richardson-Lucy deconvolution algorithms, and a new method designed to synthesize the surface response by least squares minimization. The Wiener filter is an optimal linear filter used to minimize the mean squared error between the expected surface response and the estimated surface response. ${ }^{8}$ This method $^{-}$ has been used by Jutzi and Stilla to obtain the surface response. ${ }^{5}$ Richardson-Lucy deconvolution is a nonlinear iterative technique that converges to the maximum likelihood solution for the surface response estimate. ${ }^{15,16}$ 
The surface response synthesis by least squares minimization method models a ladar pulse as the summation of scaled subpulses of the transmitted pulse that return at varying ranges depending on the surface of the target. ${ }^{1}$ If the target surface is hit at $90^{\circ}$, all subpulses return at the same time; however, if two distinct surfaces are hit, the subpulses return in two groups. When the pulse hits the surface at an angle, the subpulses are spread out and cause blurring in the return waveform. ${ }^{5}$

Surface response synthesis by least squares minimization assumes that at each time sample, there is a subpulse with an unknown amplitude ranging from 0 to the amplitude of the received waveform. The signal containing the amplitudes is the surface response estimate $\tilde{s}(n)$. If the surface response estimate is convolved with the transmitted pulse, the received waveform will result with some error $e(n)$ given by

$$
e(n)=\tilde{r}(n)-\tilde{h}(n) * \tilde{s}(n) .
$$

The error can be measured by (9) and minimized by changing the amplitudes of the subpulses using an iterative least squares technique. The technique used for least squares minimization and tested in this paper is the Levenberg-Marquardt algorithm. ${ }^{17}$

\subsection{Range discrimination}

The EM algorithm was chosen to perform Gaussian decomposition of the surface response for range discrimination. The first step in this algorithm is to estimate the number of pulses in $\tilde{s}(n)$. This is done by counting the number of peaks the signal using a first order differentiator. Only if a peak is significant with respect to a user defined threshold is it considered to be a valid return. Any signal that is disjoint from a significant peak is considered to be noise and is set to 0. This prevents the EM algorithm from fitting unwanted data to the Gaussian pulse. The estimated amplitudes, locations, and widths of the pulse(s) found by locating peaks are used to initialize the algorithm. The mean of each pulse estimated by the EM algorithm is then used to obtain the range of the pulse.

\section{EXPERIMENTS ON DECONVOLUTION TECHNIQUES}

\subsection{Range error on single surface}

Range error is a very important measure of the performance of a ladar system. This error can be measured statistically by ${ }^{1}$

$$
\sigma_{r}=\left.\frac{c}{2} \cdot \frac{\sigma_{d}\left(t_{p}\right)}{\left|\frac{d}{d t} v\left(t_{P}\right)\right|}\right|_{t=t_{p}}
$$

where

$c=$ the speed of light,

$\sigma_{d}\left(t_{p}\right)=$ the detection noise at the timing point $t_{p}$, and

$v(\cdot)=$ the pulse waveform.

This equation indicates that the range error depends greatly on the slope of the waveform at the point of detection and the noise level in the waveform. The surface response provides a signal with a higher slope, but amplified noise due to the high pass nature of deconvolution. For this reason, deconvolution techniques were tested to discover the tradeoff between increased resolution and the effects on the range error.

To test the range error, a flat, white Lambertian target with estimated reflectivity of 0.7 was constructed and placed at a range of $493 \mathrm{~m}$. The ELT was set to hold the scanner stationary while sending multiple pulses. This provided a large number of samples for analysis. The data was processed using four techniquesstandard processing without deconvolution, Richardson-Lucy deconvolution, the Wiener filter for deconvolution, and surface response synthesis by least squares minimization. The standard deviations of the range for each test were computed from the data, and are found in Table 1. 
Table 1: Range error at 493 meters.

\begin{tabular}{|c|c|}
\hline Method & Range error $(\mathrm{mm})$ \\
\hline Standard & 2.4 \\
Wiener filter & 2.5 \\
Richardson-Lucy & 3.1 \\
Least squares minimization & 2.5 \\
\hline
\end{tabular}

\subsection{Resolution of two surfaces}

To measure the ability to resolve two surfaces, the same target as in section 4.1 was again placed 493 meters from the ELT. The face of the target used for this test had a $20 \mathrm{~cm} \mathrm{X} 20 \mathrm{~cm}$ square hole cut out from the middle. Surrounding the hole, sufficient area remained on the border of the target to act as the first surface. Directly behind the hole, a second surface was placed capable of varying in range. This provided two surfaces that have a variable distance between them.

To obtain shots that hit both surfaces, the ELT scanner was set to scan across the hole in the board. From the pointcloud, 25 to 30 shots that hit both surfaces were found and processed using the different deconvolution techniques. Figure 6 shows the results of the experiment as the distance between the two surfaces varied. Fig. 6 (a) shows the difference between the hand measured distance and the distance estimated by each technique. Fig. 6 (b) is the standard deviation of the measurements. This gives a measure of the consistency of the method's estimate of the distance.

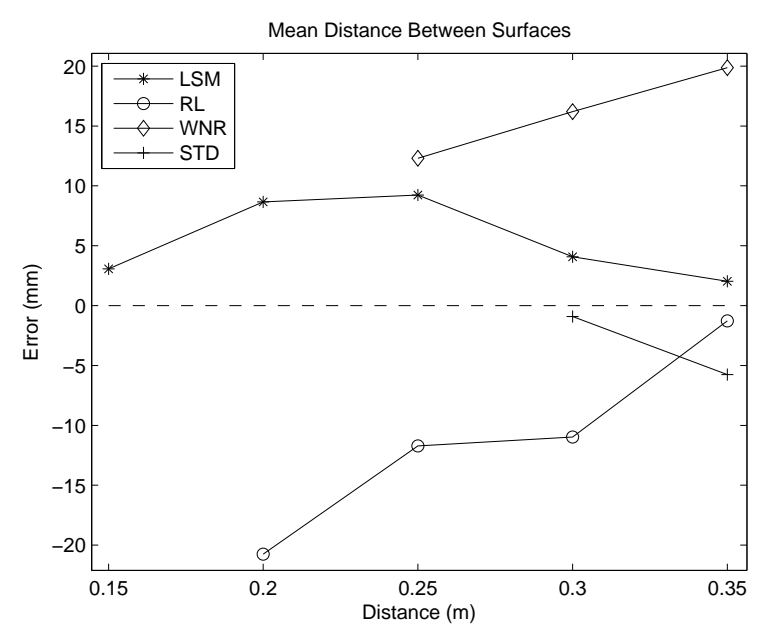

(a)

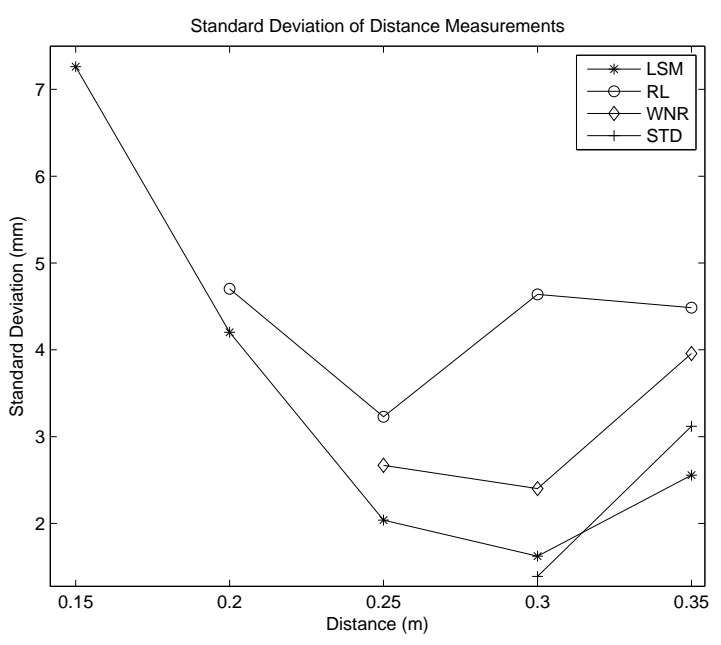

(b)

Figure 6: Measurements from return hitting two surfaces as the distance of separation between the surfaces varied. (a) Error in the calculated mean of the separation. (b) Standard deviation of the measurement.

The resolving limits of each method was also tested to the nearest centimeter. Results showing the smallest separation at which the algorithms successfully found the two peaks are shown in Table 2. Plots of example waveforms after deconvolution are shown in Fig. 7.

Table 2: Minimum distance at which two surfaces can be resolved.

\begin{tabular}{|c|c|}
\hline Method & distance $(\mathrm{cm})$ \\
\hline Standard & 29 \\
Wiener filter & 23 \\
Richardson-Lucy & 20 \\
Least squares minimization & 11 \\
\hline
\end{tabular}




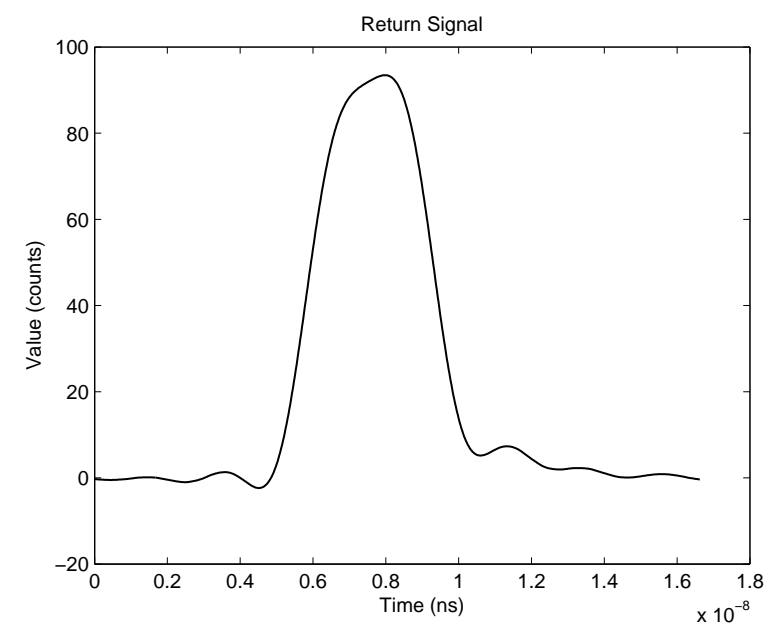

(a)

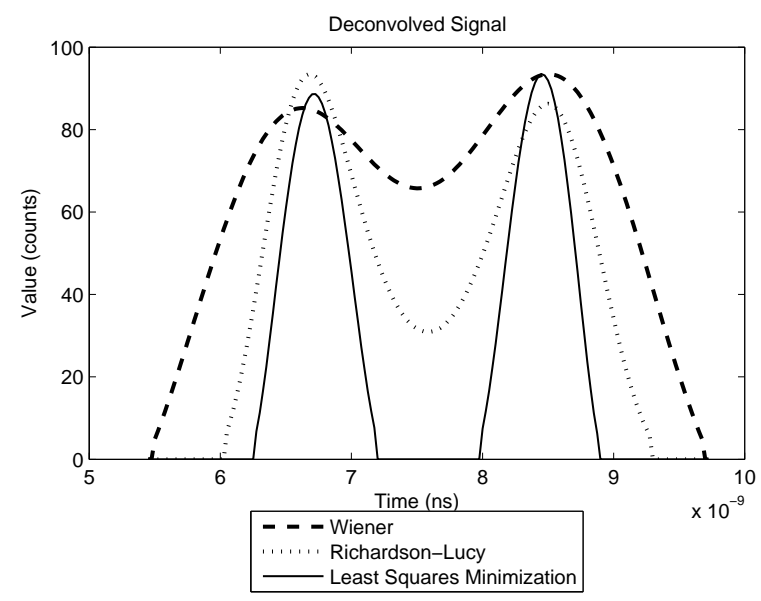

(b)

Figure 7: (a) The return waveform after hitting the two surfaces separated by $25 \mathrm{~cm}$. (b) The surface response of the same pulse using the deconvolution methods.

\section{CONCLUSION}

The test to obtain the range error using the deconvolution techniques indicates that the range error only decreased significantly for the Richardson-Lucy algorithm. The other methods decreased very slightly. Although the range error increased for all methods, deconvolution methods extract more information than the blurred received signal. The added information comes at the cost of a small increase in range error. In waveforms with a lower SNR, the tradeoff may become more significant, but was not explored in this study.

Results from section 4.2 show that surface response synthesis by least squares minimization improves the range resolution over the other methods tested. It detected two peaks at surface separations smaller than other techniques in all cases tested. The improved resolution comes with little or no tradeoff with respect to other performance metrics. The cost of surface response synthesis by least squares minimization is that it is an iterative algorithm that converges slowly. Thus, this method has the highest computational cost. The Richardson-Lucy algorithm is next at the ability to resolve close surfaces, but suffers more in range error and in the estimate of the separation. The Wiener filter is not able to detect small separations as well, but doesn't present as much tradeoff with other metrics as the Richardson-Lucy algorithm. In comparison to previous work, ${ }^{5}$ the Wiener filter was not able to deconvolve the signal to the same extent with respect to the original pulse width. The biggest contributing factor to this result is believed to be the difference in the sample rate with respect to the pulse width.

It is important to note the resolution of the surface response synthesis by least squares minimization algorithm with respect to the sample rate. First, the transmitted pulse is sampled at about two times the Nyquist frequency. Being close to the minimum sample rate, the pulse is detected with only six or seven samples that are spaced at $7.5 \mathrm{~cm}$ in range. With the deconvolution algorithm introduced in this paper, two peaks are resolvable at $11 \mathrm{~cm}$ which is well within two samples of the waveform.

The results from the experiments are encouraging for obtaining the surface response by a least squares minimization algorithm. A follow up paper is intended to provide details on the implementation as well as further testing of different surfaces such as sloped and multi-faceted surfaces. Also, the robustness of the algorithm with respect to SNR will be studied.

\section{REFERENCES}

[1] R. T. Pack, R. R. Fullmer, S. E. Budge, et al., USU LadarSIM Release 3.0 User's Manual. Utah State University/Space Dynamics Laboratory, Logan, UT, Mar. 2007. 
[2] C. Mallet and F. Bretar, "Full-waveform topographic lidar: State-of-the-art," ISPRS Journal of Photogrammetry $\mathscr{B}$ Remote Sensing 64, pp. 1 - 16, Jan. 2009.

[3] A. Neuenschwander, L. Magruder, and R. Gutierrez, "Signal processing techniques for feature extraction and classification using small-footprint full-waveform airborne LIDAR," in Proc. IEEE Int. Geoscience and Remote Sens. Symp., 3, pp. 676-679, IEEE, July 2008.

[4] W. Wagner, A. Ullrich, V. Ducic, T. Melzer, and N. Studnicka, "Gaussian decomposition and calibration of a novel small-footprint full-waveform digitising airborne laser scanner," ISPRS Journal of Photogrammetry E Remote Sensing 60, pp. 100-112, 2006.

[5] B. Jutzi and U. Stilla, "Range determination with waveform recording laser systems using a Wiener filter," ISPRS Journal of Photogrammetry \& Remote Sensing 61, pp. 95-107, 2006.

[6] J. Jung and M. M. Crawford, "A two-stage approach for decomposition of ICESat waveforms," Proc. IEEE Int. Geoscience and Remote Sens. Symp. 3, pp. 680-683, 2008.

[7] N. Vlassis and A. Likas, "A greedy EM algorithm for Gaussian mixture learning," Neural Processing Letters 15, pp. 77-87, 2002.

[8] T. K. Moon and W. C. Stirling, Mathematical Methods and Algorithms for Signal Processing, ch. 17, pp. 717743. Prentice Hall, Upper Saddle River, NJ 07458, 2000.

[9] B. Jutzi, J. Neulist, and U. Stilla, "Sub-pixel edge localization based on laser waveform analysis," in International Archives of Photogrammetry, Remote Sensing and Spatial Information Sciences, 36(Part /W8), pp. 109-114, JSPRS, 2005.

[10] M. Kirchhof, B. Jutzi, and U. Stilla, "Iterative processing of laser scanning data by full waveform analysis," ISPRS Journal of Photogrammetry \& Remote Sensing 63, pp. 99-114, 2008.

[11] R. T. Pack, J. A. Swasey, R. R. Fullmer, S. E. Budge, P. D. Israelsen, B. Petersen, and T. D. Cook, "Eyesafe LADAR test-bed with coaxial color imager," in Laser Radar Technology and Applications XIV, M. D. Turner and G. W. Kamerman, eds., 7323, SPIE, Apr. 2009.

[12] R. T. Pack, P. Israelsen, and K. Sealy, "A co-boresighted synchronized ladar/EO imager for creating 3D images of dynamic scenes," in Laser Radar Technology and Applications X, G. W. Kamerman, ed., 5791, pp. 42-50, SPIE, May 2005.

[13] K. D. Neilsen, S. E. Budge, R. T. Pack, R. R. Fullmer, and T. D. Cook, "Design and validation of the eyesafe LADAR test-bed (ELT) using the LadarSIM system simulator," in Laser Radar Technology and Applications XIV, M. D. Turner and G. W. Kamerman, eds., 7323, pp. 73230B-1-73230B-7, SPIE, May 2009.

[14] J. G. Proakis and D. G. Monolakis, Digital Signal Processing, ch. 11, pp. 760-762. Prentice Hall, Upper Saddle River, NJ 07458, 2007.

[15] W. H. Richardson, "Bayesian-based iterative method of image restoration," J. Opt. Soc. Amer. 62, pp. 5559, Jan. 1972.

[16] L. B. Lucy, "An iterative technique for the rectification of observed distributions," The Astronomical Journal 79(6), pp. 745-754, 1974.

[17] D. Marquardt, "An algorithm for least-squares estimation of nonlinear parameters," Journal of the Society for Industrial and Applied Mathematics 11, pp. 431-441, June 1963. 